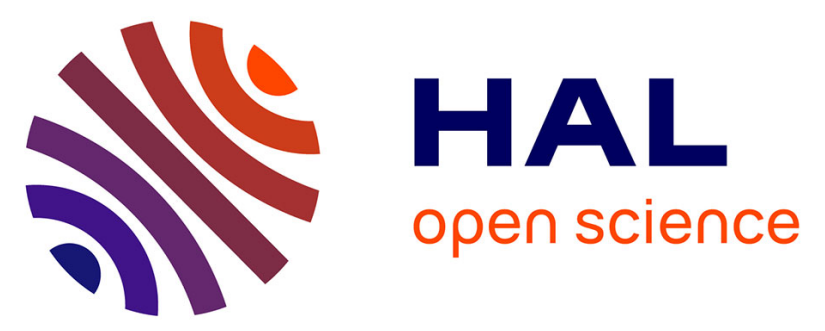

\title{
Methods for a Quantitative Comparison of and Posture Control Over a Wide Range of Herbaceous and Woody Species
}

Félix Hartmann, Hugo Chauvet-Thiry, Jérôme Franchel, Stéphane Ploquin, Bruno Moulia, Nathalie Leblanc-Fournier, Mélanie Decourteix

\section{To cite this version:}

Félix Hartmann, Hugo Chauvet-Thiry, Jérôme Franchel, Stéphane Ploquin, Bruno Moulia, et al.. Methods for a Quantitative Comparison of and Posture Control Over a Wide Range of Herbaceous and Woody Species. Elison B. Blancaflor. Plant Gravitropism, 2368, Springer US, pp.117-131, 2021, Methods in Molecular Biology, 978-1-0716-1677-2. 10.1007/978-1-0716-1677-2_9 . hal-03400916

\section{HAL Id: hal-03400916 https: / hal.inrae.fr/hal-03400916}

Submitted on 5 Nov 2021

HAL is a multi-disciplinary open access archive for the deposit and dissemination of scientific research documents, whether they are published or not. The documents may come from teaching and research institutions in France or abroad, or from public or private research centers.
L'archive ouverte pluridisciplinaire HAL, est destinée au dépôt et à la diffusion de documents scientifiques de niveau recherche, publiés ou non, émanant des établissements d'enseignement et de recherche français ou étrangers, des laboratoires publics ou privés. 


\title{
Methods for a quantitative comparison of gravitropism and posture control over a wide range of herbaceous and woody species
}

\author{
Félix P. Hartmannn ${ }^{1,4}$, Hugo Chauvet-Thiry ${ }^{2,3,4}$, Jérôme Franchel ${ }^{1}$, Stéphane Ploquin ${ }^{1}$, Bruno Moulia ${ }^{1}$, \\ Nathalie Leblanc-Fournier ${ }^{1,5}$, Mélanie Decourteix ${ }^{1,5}$ \\ ${ }^{1}$ Université Clermont Auvergne, INRAE, PIAF, F-63000 Clermont-Ferrand, France \\ ${ }^{2}$ Aix-Marseille Université, CNRS, IUSTI, Marseille, France \\ ${ }^{3}$ DISCO beamline, Synchrotron Soleil, L'Orme des merisiers, Gif-sur-Yvette, France \\ ${ }^{4}$ These authors contributed equally to the work \\ ${ }^{5}$ These authors contributed equally to the work \\ Contact address: felix.hartmann@inrae.fr
}

\begin{abstract}
Quantitative measurements of plant gravitropic response are challenging. Differences in growth rates between species and environmental conditions make it difficult to compare the intrinsic gravitropic responses of different plants. In addition, the bending movement associated with gravitropism is competing with the tendency of plants to grow straight, through a mechanism called proprioception (ability to sense its own shape). Disentangling these two tendencies is not trivial. Here, we use a combination of modeling, experiment and image analysis to estimate the intrinsic gravitropic and proprioceptive sensitivities of stems, taking Arabidopsis as an example.
\end{abstract}

Keywords: Kinematics, Proprioception, Posture control, Plantlets, Image analysis

\section{Introduction}

Interest for how plants maintain a straight and erected growth habit led plant biologists to focus on tropisms, the orientation of growth in the direction of an environmental stimulus depending on the direction of the stimulus. Major progresses have thus been gained in the study of gravitropism and phototropism.

Plant gravitropism entails slow growth movement allowing a reorientation of organs relative to the gravity field. Despite a growing interest for such movements and the recent insights into the molecular mechanisms of gravitropic sensing (see (1) for a recent review), little is known about its quantitative biological control. In plants, gravisensing relies on small starch-filled organelles called statoliths. When a plant is leaned, their sedimentation in the direction of the gravity field triggers asymmetric growth and, consequently, curvature of the organ. For a long time, this growth response has been interpreted as a consequence of the perception of the weight of statoliths and/or of the protoplast, meaning that these structures would behave as force sensors. Our research team recently developed an original device enabling us to (i) study the effect of the direction (i.e. the angle between the initial inclination of the plant shoot and the gravity vector) vs the intensity of the gravity vector for the study of long-term stimuli (2), or (ii) realize gravity dose-response studies in the context of transient stimulations (3). Thanks to this device, (2) dismissed the statolith weight and protoplast pressure models in aerial organs by demonstrating that shoot gravitropism relies on the sensing of the inclination and not on the sensing of gravitational force or acceleration. They thus 
validated the other long-standing model of gravitropic control based on the sensing of the inclination angle with respect to the gravity field. It would thus seem logical to continue to focus on measures related to gravitropism with well-proven techniques consisting of following the tip angle of organs. However, in the last decade, studies based on kinematic analyses combined with mathematical modeling highlighted that the gravitropic response cannot be explained by the sensing of the inclination angle only (4). Such approaches revealed the importance of the straightening movement in aerial organs. So, to preserve their erected habit and grow straight, plant aerial organs must have the ability to sense their inclination with respect to the gravity field (graviception) as well

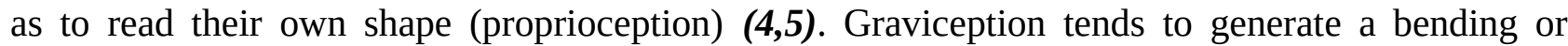
curving movement upward toward the vertical while proprioception tends to rectify the organ by decreasing its curvature. To understand the gravitropic uprighting process and to separate the part of the response due to graviception from the part due to proprioception, it thus appeared necessary to not only measure the inclination of the organ but also to monitor the local curvature $C$ over the entire growth zone.

How to measure a curvature and what are the underlying biological concepts? The curvature $C$ can be defined as the spatial rate of change of the angle $A$ along the organ $(6,7)$ :

$C=\frac{\partial A}{\partial s}$

$s$ is the spatial coordinate along the organ and $\frac{\partial A}{\partial s}$ is the derivative of $A$ versus spatial position (partial derivative). When $C=0$ everywhere, the organ is straight. During a tropic response, the curvature changes locally with time. To properly quantify the response of an organ, it is useful to describe the growth mechanism at the origin of the curvature changes. Any change in curvature results from differential growth, i.e. a difference between the elongation rate of the lower face $\left(\dot{\varepsilon}_{\text {low }}\right)$ and the elongation rate of the upper face ( $\dot{\varepsilon}_{\text {up }}$ ) of the organ. For instance, if the lower face elongates faster than the upper face, the organ curves upward. Locally, curvature variation is directly driven by the difference in elongation rates:

$\frac{D C}{D t} R=\frac{\dot{\varepsilon}_{\text {low }}-\dot{\varepsilon}_{\text {up }}}{2}$

where $R$ is the radius of the organ and $\frac{D C}{D t}$ is the time derivative of the curvature (curving rate) attached to each successive segment (i.e. material derivative, see (6) for a further biologist-friendly priming on the quantification of tropic response including material derivatives).

However, these relative elongation rates depend a lot on growth conditions and can vary from one species to another. The effect of such variations on the computation of curvature changes can be taken into account by considering the mean relative elongation rate $\dot{\varepsilon}_{\text {mean }}$ defined as:

$\dot{\varepsilon}_{\text {mean }}=\frac{\dot{\varepsilon}_{\text {low }}+\dot{\varepsilon}_{\text {up }}}{2}$

Then, equation (1) can be re-written as:

$\frac{D C}{D t}=\dot{\epsilon}_{\text {mean }} \tilde{\Delta}$

where $\tilde{\Delta}$ is the differential growth ratio and is defined as:

$\widetilde{\Delta}=\frac{\dot{\varepsilon}_{\text {low }}-\dot{\varepsilon}_{\text {up }}}{\dot{\varepsilon}_{\text {low }}+\dot{\varepsilon}_{\text {up }}}$ 
The interest of $\tilde{\Delta}$ is that it measures specifically the redistribution of the mean growth toward the lower and upper faces.

Now that a way to assess curvature has been provided, it is important to understand how graviception and proprioception contribute to this curvature. To this end, it is necessary to assess the graviceptive and proprioceptive sensitivities of the organ. (8) provided a mathematical model, the $A C \dot{E}$ model, enabling to assess these sensibilities. It assumes that the relative differential growth $\tilde{\Delta}$ is a local response to the inclination angle $A$ (graviception) and to the curvature $C$ (proprioception):

$\widetilde{\Delta}=-\tilde{\beta} A-\tilde{\gamma} C R$

$\widetilde{\beta}$ and $\tilde{\gamma}$ are respectively the gravitropic and proprioceptive sensitivities. The tilde symbols indicate that these parameters are dimensionless and normalized for the mean elongation rate and the radius, in contrast with the previous formulation in (4). This makes it possible to directly compare the sensitivities of two different plants, independently of their size and mean elongation rate.

Note that $A, C$ and $\tilde{\Delta}$ are functions of the position along the organ $(s)$ and of time $(t)$. The organ radius $R$ is assumed constant and uniform along the organ. The mean elongation rate $\dot{\varepsilon}_{\text {mean }}$ is assumed uniform along the growth zone and constant in time. $\widetilde{\beta}$ and $\tilde{\gamma}$ are intrinsic parameters of the plant and depend on neither space nor time.

Once the organ has reached a steady-state (i.e. its shape does no longer depend on time), the inclination angle along the growth zone is given by:

$A_{s t}(s)=A_{0} \exp \left(-B \frac{s}{L_{g z}}\right)$

$A_{0}$ is the angle at the base of the growth zone, $L_{g z}$ is the length of the growth zone and $B$ is the dimensionless "balance number" expressed as:

$B=\frac{\tilde{\beta} L_{g z}}{\tilde{\gamma} R}$

$B$ is an important integrative trait which combines the gravitropic and proprioceptive sensitivities with the geometry of the organ (aspect ratio of the growth zone $L_{g z} / R$ ). It determines both the final shape of the organ and the time required to reach this shape (see details in (7)).

In this chapter, we describe how to monitor the kinematics of the gravitropic response (inclination and curvature), and how to estimate the gravitropic and proprioceptive sensitivities through experiment and semi-automatic image analysis, with the help of a software (Interekt) which has been developed in our laboratory. We hereafter describe our method by taking Arabidopsis as an example, but it can be adapted to other species.

\section{Materials}

\section{Experiment}

1. Seeds (Arabidopsis thaliana, Columbia-0 accession) (Notes 1 and 2)

2. Cylindrical containers to grow the plants. We use Polypropylene sample container (30 ml)

3. Potted soil added with fertilizing media

4. Growth chamber or growth room

5. Refrigerator to vernalize the seeds

6. A system to clamp pots horizontally ahead a dark background. In our case, it consists of a medium-density fiberboard which was painted in black. Fixing collars ( $3 \mathrm{~cm}$ diameter) were 
attached to this board allowing to clamp Arabidopsis plants (grown in cylindrical containers) horizontally. To avoid phototropism effects, this device has to be placed in a dark room.

7. A camera allowing time-lapse imaging of plant gravitropic kinematics. The camera must possess a wide-angle lens (for example, NIKON D200). To avoid phototropism effects, pictures have to be taken in the dark. Thus, the flash must be filtered in the green safelight waveband.

\section{Software}

Interekt (Interactive Exploration of Kinematics and Tropisms) is an open-source software devoted to the quantitative analysis of one-dimensional organ growth. It is written in the programming language Python and depends on several Python libraries. It has been tested on Linux, Windows and

OS X. The version of Interekt used in this chapter, along with installation guidelines, is available online (https://forgemia.inra.fr/hugo.chauvet-thiry/rootstemextractor/-/tree/chapter gravitropism).

\section{Methods}

\section{Experiment}

1. Vernalize the seeds at $4^{\circ} \mathrm{C}$ for at least three days.

2. Fill-up cylindrical containers with potted soil added with fertilizing media. Sample containers can be pierced with holes to water the plantlets by sub-irrigation before the imaging process.

3. Dispense one seed per container.

4. Grow Arabidopsis plants in a growth chamber until inflorescences are 6-8cm tall. Plants will be ready for analysis after about 6 weeks in the growth chamber under typical conditions $\left(16 \mathrm{~h} / 8 \mathrm{~h}\right.$ day/night cycle; $23^{\circ} \mathrm{C}$ during the day and $19^{\circ} \mathrm{C}$ during the night; $50 \%$ air hygrometry). Water the plants regularly by sub-irrigation.

5. Once the plants have reached the required stage, and the day before leaning the plant (Note 3):

- Cover the rosette leaves with a net. This step is necessary because when plants are leant horizontally, leaves can become hyponastic and mask the lower part of the floral stem. Alternatively, attach the rosette leaves to the cylindrical container with pieces of tape.

- In order to ease the digital (automatic) tracking of the inflorescence movements, it is better to work with a single unbranched floral stem. For this purpose, remove all the inflorescence ramifications and cauline leaves with a scalpel (Note 3).

6. A few hours before leaning the plant, position a black mark with a permanent marker at the tip of the inflorescence, at the bottom of the terminal group of flowers. This mark will be used by the Interekt software to track the movements of the floral stem (Note 4).

7. Clamp the container horizontally ahead a dark background (see Material).

8. Program camera time-lapse: 1 photo every 6 minutes during the first and fastest part of the gravitropic movement (about two hours), and a final photo after 36 hours to have the final shape of the stem (this will be the steady-state image).

9. Start picture acquisition on the camera.

\section{Image analysis and semi-automatic estimation of important parameters}

1. Import the sequence of images generated from the experiment by clicking on the 'Open' button in the toolbar and then selecting images. The software automatically stores the images into a single archive file (see Note 5). This can take some time. After this operation is completed, the first image of the sequence is displayed in the main window. 
2. If you wish, inspect visually the experiment by browsing through the sequence. Press the right (resp. left) arrow key to move forward (resp. backward) in the sequence. Alternatively, click on the 'Image list' button in the toolbar to open a small floating window, from which you can directly access a given image by clicking on its name.

3. Select the steady-state image (i.e. the image at which you consider the organ has reached its final shape; usually the last image of the sequence). Click on "Options" and then "Select stead-state image”. A popup window appears, with the list of images (Fig. 1). Click on the image you consider at steady state. You have the possibility to exclude this image from time series by checking the box below the list. This can be useful when the steady-state image has been taken a long time after other images.

4. The image scale can be set with the "Scale" window (Options $\rightarrow$ Scale). Click on "Measure distance". Then, choose two points of the current image separated by a known distance. Click successively on these two points. A line joining the points appears, and the "pixels" field of the "Scale" window is automatically filled in with the number of pixels between the points. Fill in the "cm" field with the distance in centimeters between the points. Then click on "Update the scale" and close the "Scale" window. All distances in pixels will be converted into cm using this ratio (see Note 6).

5. For extracting organ shapes, move to the first image of the sequence and click on the "Add a base" button in the toolbar. A "base" is an object which indicates the beginning of the organ and its direction. To create the base, left-click successively on both sides of the organ (each time outside the organ), close to the anatomical base of the organ. A line segment crossing the organ appears, with a shorter line segment orthogonal to the main segment. This orthogonal line segment indicates the direction which will be followed by the algorithm extracting the organ shape. It depends on the order in which the sides of the organ are clicked when creating the base. For an organ growing from left to right, click first above and then below the organ (and inversely for an organ growing from right to left). For an organ growing from top to bottom, click first right and then left of the organ (and inversely for an organ growing from bottom to top).

6. Once a base has been created on each organ to analyze, click on the "Treat" button in the toolbar. This triggers organ shape extraction and may take some time. At the end of the treatment, extracted organ skeletons appear as colored lines on the organs (Fig. 2). If you want to give a specific label to the organ, see Note 7 . If the shape extraction ends up with an incorrect skeleton, see Note 8 .

7. Geometric quantities can be computed from the skeleton of an organ for the current image. They can be visualized by right-clicking on a skeleton and selecting "Angles and curvatures” from the context menu. A new window pops up, with three plots. The topmost plot shows the profile of the organ, which is nothing more than its extracted skeleton. The middle plot shows the inclination (angle from vertical) along the organ. The bottom plot shows the curvature along the organ.

8. It is possible to get an overlook of the kinematics of an organ, and thus a first insight into possible tropisms, by right-clicking on a skeleton and selecting "Time series" from the context menu (or, alternatively, simply by left-clicking on the skeleton). A new window pops up, with three plots (Fig. 3). The plot from the left is made of the superimposition of the profiles of the organ during the experiment. The successive profiles are color-coded using a colormap ranging from purple (for the beginning of the experiment) to yellow (for the end of the experiment). The steady-state profile is colored separately in red. The topright plot represents the tip angle over time. The bottom-right graph represents the length of the organ over time. If you had decided to exclude the steady state from time series, it does not appear in these two last graphs. 
9. For estimating the growth rate of an organ $(\mathrm{d} L / \mathrm{d} t)$, right-click on its skeleton and select "Estimate growth rate" from the context menu. A new window pops up, with four plots. The plot from the left is the same superimposition of organ profiles as explained above ("Time series"). The top-right plot shows the growth curve of the organ (length as a function of time). The growth rate is estimated through a linearization of the growth curve, or at least a part of it. On this top-right plot, you can select the part of the curve which is closest to linearity. Left-click on the plot and move the mouse pointer while clicking to draw a rectangle on the zone you want to select (see Note 9).

10. The middle-right plot of the "Growth rate" popup window shows the growth length of the organ, zoomed-in according to the rectangle drawn on the plot above. In addition, there are two red dots joined by a line segment. By moving the dots with the mouse (left click), you can position the line segment such that it represents the linearization of the growth curve. The growth rate $\mathrm{d} L / \mathrm{d} t$ (computed from the slope of the line) is displayed on the left plot. The bottom-right plot shows the residuals of the linearization. This gives a way to estimate the goodness of the linearization.

11. For estimating the growth length $L_{g z}$ of an organ, right-click on its skeleton and select "Estimate growth length" from the context menu. A new window pops up, with three plots. The bottom plot represents the organ curvature across space and time using a heatmap. Space goes along the x-axis, while time goes along the $y$-axis. Warm colors (yellow, red, dark red) code for positive curvatures, while cold colors (blue, dark blue) code for negative curvatures. On the right edge, a solid black line represents the linearized growth as estimated above. A draggable dotted red line parallel to the linearized growth enables you to select the growth length, or more exactly the boundary between the non-growing zone and the growing zone of the organ (see Note 10). During gravitropic movement, differential growth is strong, which means that the curvature is changing with time everywhere in the growth zone. This is how growth can be spotted: Where curvature does not change along the $\mathrm{y}$-axis (time), there is no growth, whereas a curvature change along the $y$-axis signals growth. A horizontal draggable dotted line provides an auxiliary tool for detecting the boundary of the growing zone. Dragging this line sets the current time, with an effect on both top plots. The top-left plot shows the angle along the organ, at the initial time and at the current time. Comparing the angles at two different times can help spotting where growth occurs. The top-right plot pictures the organ in its initial state, with the superimposition of the organ skeleton at the current time. The big red dot on the image represents the position of the vertical draggable line. This superimposition offers a third way to check the estimation of the growth length. The numerical values of the estimated growth length $L_{g z}$ is displayed at the bottom of the window.

12. For estimating the parameter $\widetilde{\beta}$ of an organ, right-click on its skeleton and select "Estimate beta”. A new window pops up, with three plots (Fig. 4). The left plot shows the successive profiles of the organ, as already described. The top-right plot represents the tip angle over time. You need to make a linear regression on this curve to estimate $\widetilde{\beta}$. Two vertical blue draggable lines allow you to select the regression range. The $R^{2}$ of the fit is displayed on the plot. The estimated value of $\widetilde{\beta}$ is displayed on the left plot, along with quantities involved in the estimation: The radius $R$, the initial tip angle $A_{\text {init }}$, the rate of angle change $\mathrm{d} A / \mathrm{d} t$ (obtained from the linear regression), and the growth rate $\mathrm{d} L / \mathrm{d} t$ (see Note 11 ). The bottomright plot represents the growth, with its linearization, as a reminder.

13. For estimating the balance number $B$ of an organ, right-click on its skeleton and select “Estimate B". A new window pops up, with three plots (Fig. 5). The top-left plot represents the inclination angle along the growth zone, for the profile selected before as steady state. The top-right plot show the steady-state image of the organ, with the extracted skeleton. The bottom plot represents the normalized inclination angle $A / A_{0}$ along the growth zone (where 
$A_{0}$ is the angle at the base of the growth zone). This curved is fitted with an exponential (red dotted line, see Note 12). No action is required from the user; the regression is automatic. If you are satisfied with the fit, just close the popup window. Otherwise, you can try selecting another image as steady state and/or re-estimating the growth length.

14. As a last step, export the results into an output CSV file. Click on "Export" and then "Phenotype (graviception, proprioception)". The resulting CSV file contains all previously estimated values, plus the proprioceptive parameter $\tilde{\gamma}$ (see Note 13).

\section{Notes}

1. In our hands, the method works well with several plants (8) (see (2) for more details on culture protocols) such as :

- wheat coleoptiles (Triticum aestivum) (between 1.5 and $2.5 \mathrm{~cm}$ tall-about 4 days after germination) originating from seeds that were germinated on cotton (for more information about a method to obtain the coleoptile, see (2))

- sunflower hypocotyls (Helianthus annuus cv Germline) (between 2 and $4 \mathrm{~cm}$ tall) originating from seeds that were germinated on soil

\section{- For taller plants and/or plants having a long curving phase: Isotropic light spheres}

In this subsection, we provide a description of a device which was first developed in our lab to disentangle gravitropism and phototropism (Coutand et al., 2019). It has recently been modified and it can now be used in studies aiming at disentangling graviperception and proprioception. In our hands, it works well with young trees (one-year old) and we gained a good command with poplar.

The uprighting and recovery movements of secondary growing plants (e.g. young trees) can be slow (several weeks for poplar) compared to those of small plants or organs displaying mainly primary growth. It then becomes necessary to maintain day-night cycles for several weeks, and it is therefore not possible to analyze these movements in the dark as is practiced in the protocol described above. However, under anisotropic lighting conditions and outside microgravity conditions (spaceflight for example), it is difficult to clearly separate gravitropism from phototropism. Indeed, when a plant is tilted, one side is lit while the other is shaded, which can induce a phototropic response. To overcome this problem, the use of an isotropic lighting device allows to tilt young trees in an isotropic light environment. It thus triggers a response devoid of its phototropic component. In our case, this device consists of two hemispheres $(1.5 \mathrm{~m}$ diameter) mounted on two metallic and hexagonal tracks. In order to increase the isotropy of the light (i) hemisphere are made of transparent polymethyl methacrylate so that lighting LED tubes can be positioned outside the hemisphere, (ii) the inside of each hemisphere has been painted in white so as to obtain a good diffusion of light inside the sphere, (iii) LED tubes were placed according to Den Dulk's 'TURTLE' pattern (Dulk, 1989). The isotropic spheres were first equipped with fluorescent tubes as a lighting system (Coutand et al., 2019). However, to limit overheating inside the sphere, they were recently replaced by LED tubes. Despite the use of LEDs, the system produces a lot of heat. Thus, to maintain the temperature at approximately $25^{\circ} \mathrm{C}$ during the lighted periods, a mobile cooling system (Blyss WAP 267EC) was connected to the sphere.

2. Plants respond to rosette leaves manipulation and to removing the ramifications and cauline leaves of the inflorescence by transiently stopping growth (a process named 
thigmomorphogenesis) (11). Growth comes back to normal several hours after these two manipulations. This is the reason why they are carried out the day before leaning the plant.

3. The black mark is placed in a growing zone. Avoid placing the mark too soon on the inflorescence unless growth will stretch the mark and reduce its intensity. This reduced intensity prevents the proper detection of the mark by the Interekt software. The mark isn't placed right before leaning the plant because of the thigmomorphogenetic Touch response (see Note 3).

4. Once a sequence of images from an experiment has been imported into Interekt, they are automatically archived into a single hdf5 file, saved in the same folder as the images, with the default name 'interekt_data.h5'. Any data obtained from subsequent processes and analyses will be automatically saved in the same archive file. Therefore, this file contains every data from the experiment. It can be opened directly from Interekt (using the 'Open' button) for any future use, which is much faster than reopening the original sequence of images.

5. The scale is the same for all images of a sequence. Make sure that the photographs were taken from the same viewpoint, with same angle and settings.

6. By default, each organ is labeled with a number. You may prefer to use a more informative label, for example with the date of the experiment and/or the name of the mutant. To do that, right-click on the skeleton, select "Settings", and then fill in the label field in the popup window.

7. If the skeleton extraction algorithm did not stop at the black mark before the inflorescence, you can try changing the threshold sensitivity. Right-click on the skeleton and select "Settings". If, for some images, the algorithm missed the mark and included the inflorescence, try decreasing the threshold sensitivity. If, conversely, the algorithm did not reach the mark, try increasing the sensitivity. Then close the "Settings" window and click again on "Treat". Tune iteratively the sensitivity until you are satisfied with the extracted skeleton.

8. Once a rectangle selector has been drawn, you can resize it using the square handlers at the corners and in the middle of each edge. You can also move the rectangle using the central handler.

9. The length of the growing zone (the growth length $L_{g z}$ ) is assumed constant through the experiment. This is why the boundary between the non-growing zone and the growing zone is oblique on the curvature heatmap and parallel to the linearized growth.

10. The exact formula is $\widetilde{\beta}=\frac{R}{\sin \left(A_{\text {init }}\right)} \times \frac{d A / d t}{d L / d t}$. The radius $R$ is averaged along the organ.

11. At steady-state, the inclination angle along the growth zone is given by: $A_{s t}(s)=A_{0} \exp \left(-B \frac{s}{L_{g z}}\right)$

12. Knowing the value of the growth length $L_{g z}$ (estimated before), an exponential fit on the inclination angle gives the value of $\mathrm{B}$.

13. The "balance number" $B$ is related to the gravi- and proprio-ceptive parameters through the relation: $B=\frac{\widetilde{\beta} L_{g z}}{\tilde{\gamma} R}$. From the values of $B, \tilde{\beta}, L_{g z}$ and $R$, we can derive the value of $\tilde{\gamma}$. 


\section{Figures}

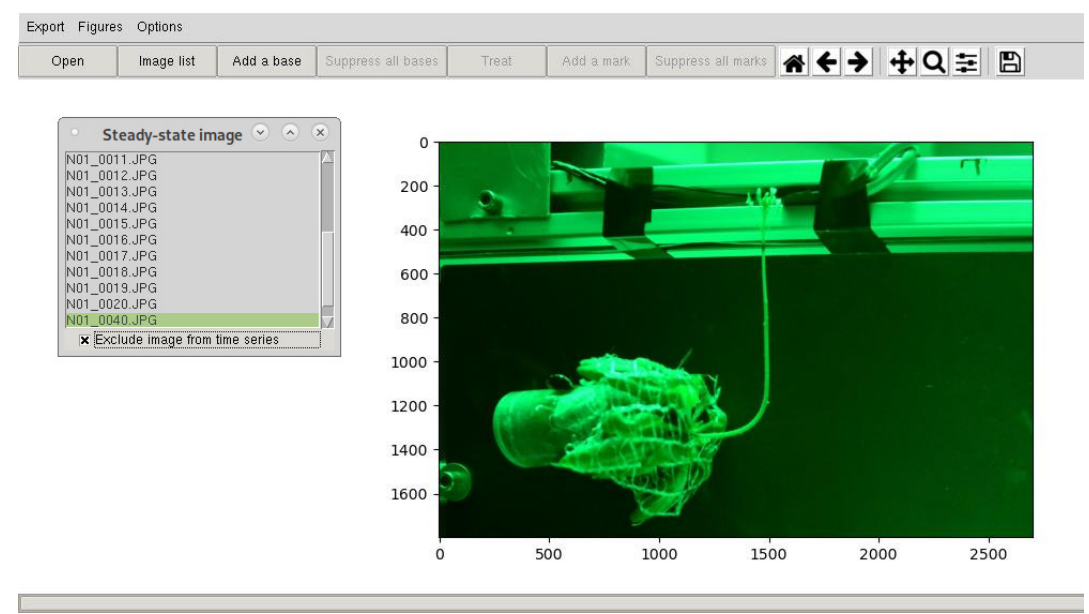

Export Figures Options

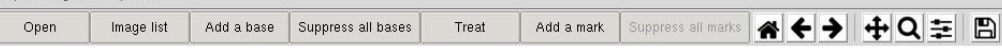
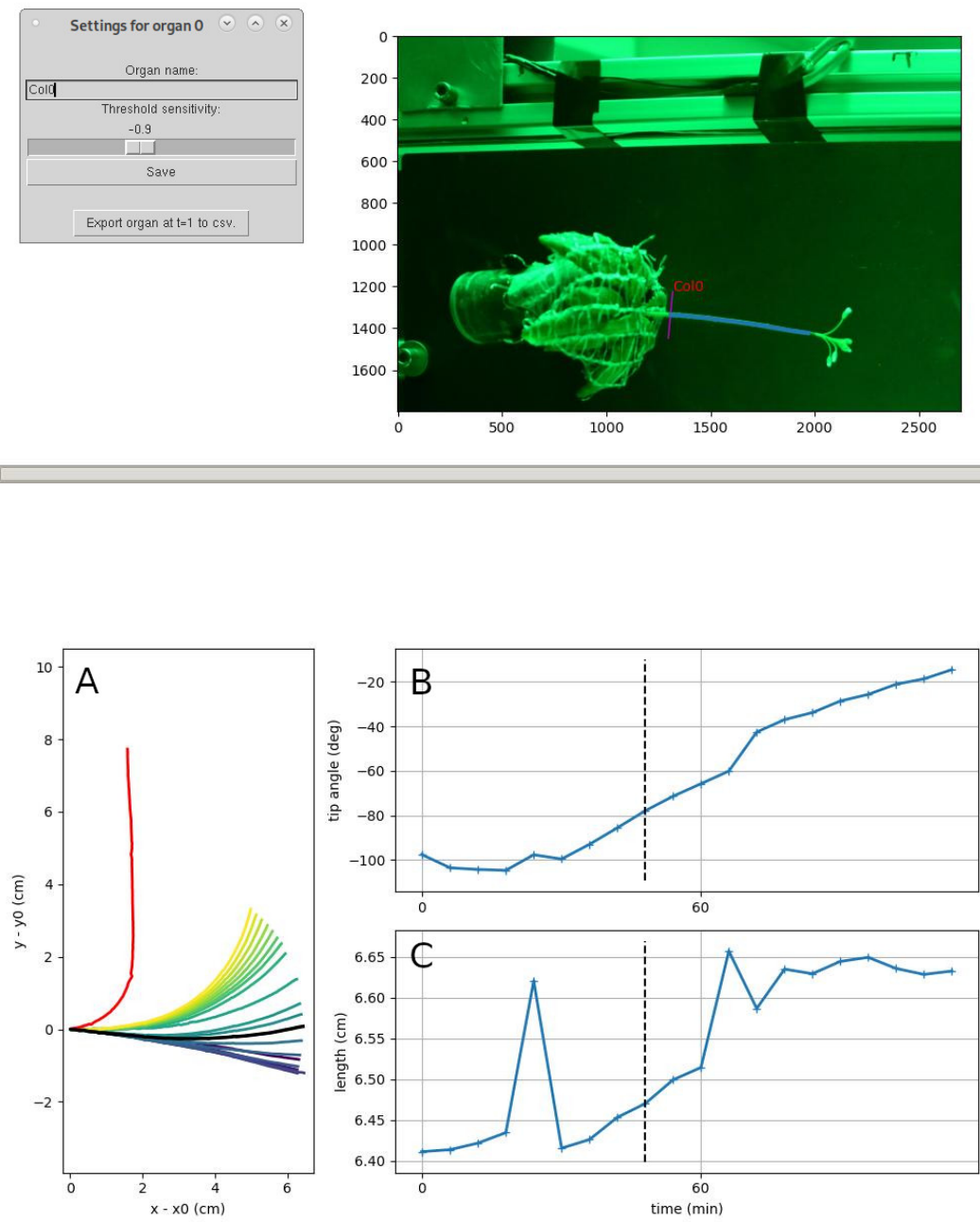

Fig. 1 Interekt interface after images have been loaded.

Selection of the steady-state image.
Fig. 2 Interekt interface after skeleton extraction of the stem. The threshold sensitivity has been set to -0.9 .
Fig. 3 Kinematic description of the tropic movement, On the time series, the vertical dotted line represents the current image. On the left plot, the steady-state profile is in red and the current profile is in black. You can see that the growth curve (length over time) is a bit irregular, due to imperfections in skeleton extraction. 

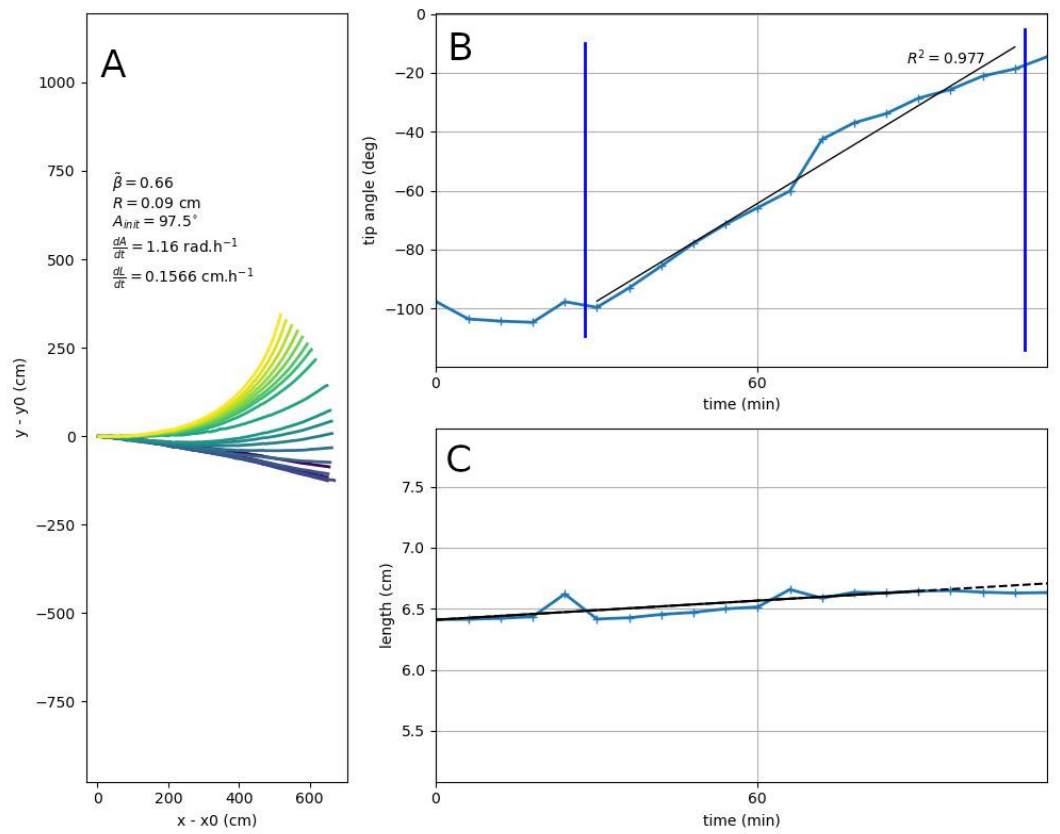

수十正回

$x=100.601 \quad y=-93.0519$

Fig. 4 Interactive estimation of $\widetilde{\beta}$.
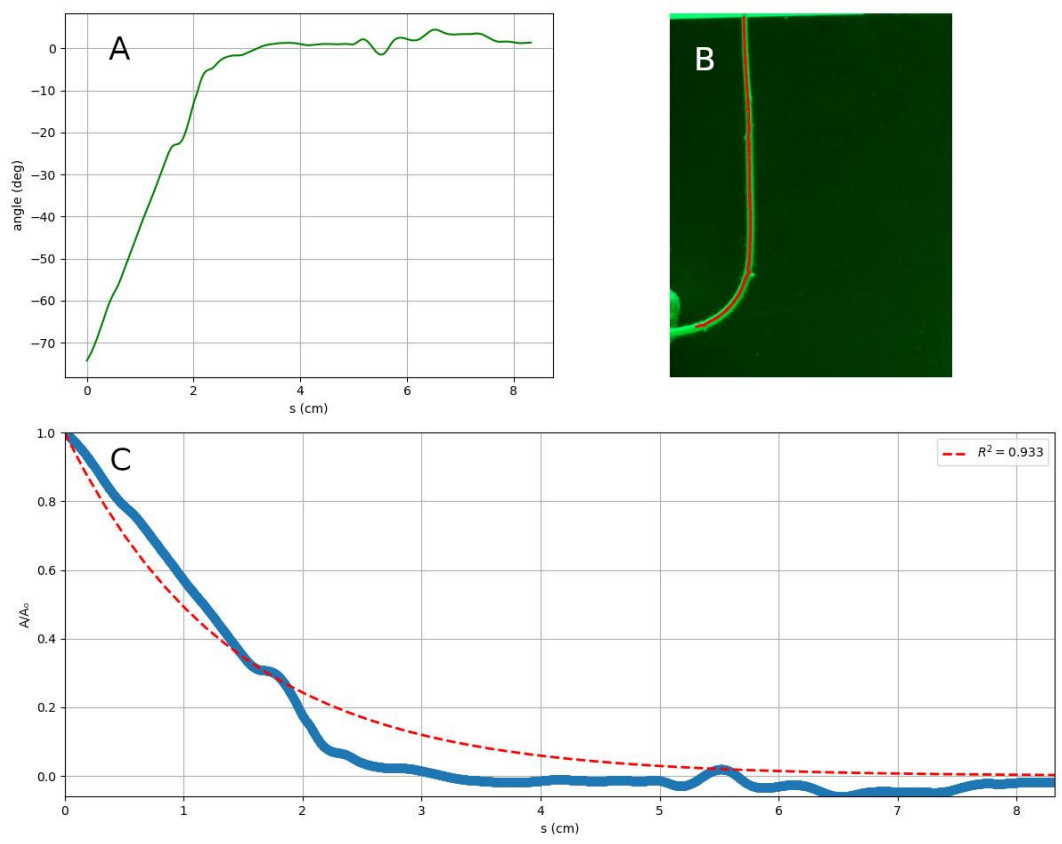

$\leqslant \leftarrow+Q=$ 回

Fig. 5 Estimation of B. 


\section{References}

1. Nakamura M, Nishimura T, Morita MT. (2019) Gravity sensing and signal conversion in plant gravitropism. Journal of Experimental Botany 70(14): 3495-3506.

2. Chauvet H, Pouliquen O, Forterre Y, Legué V, Moulia B (2016) Inclination not force is sensed by plants during shoot gravitropism. Scientific Reports 6, 35431.

3. Chauvet H, Moulia B, Legué V, Forterre Y, Pouliquen O (2019) Revealing the hierarchy of processes and timescales that control the tropic response of shoots to gravi-stimulations. Journal of Experimental Botany, Vol 70, Issue 6, 1955-1967.

4. Bastien R, Bohr T, Moulia B, Douady S (2013) Unifying model of shoot gravitropism reveals proprioception as a central feature of posture control in plants. PNAS 110, 755-760.

5. Hamant O, Moulia B. (2016) How do plants read their own shapes? New Phytologist, 212, 333-337.

6. Moulia B, Fournier M. (2009) The power and control of gravitropic movements in plants: a biomechanical and systems biology view. Journal of Experimental Botany, 60, 461-486.

7. Moulia B, Bastien R, Chauvet-Thiry H, Leblanc-Fournier N. (2019) Posture control in land plants: growth, position sensing, proprioception, balance, and elasticity. Journal of Experimental Botany, 70, 3467-3494.

8. Bastien R, Bohr T, Moulia B, Douady S (2014) A unifying modeling of plant shoot gravitropism with an explicit account of the effects of growth. Frontiers in Plant Science, 5, 136.

9. Coutand C, Adam B, Ploquin S, Moulia B. (2019) A method for the quantification of phototropic and gravitropic sensitivities of plants combining an original experimental device with model-assisted phenotyping: Exploratory test of the method on three hardwood tree species. PLOS ONE 14.

10. Den Dulk JA (1989) The interpretation of remote sensing, a feasibility study. Ph.D. Thesis, Wageningen University.

11. Chehab EW, Eich E, Braam J (2009) Thigmomorphogenesis: a complex plant response to mechanostimulation. Journal of Experimental Botany, Vol 60, Issue 1, 43-56. 